УДК $519.853+517.988 .523$

\title{
КРИТЕРИЙ СУЩЕСТВОВАНИЯ СОХРАНЯЮЩИХ УПРАВЛЕНИЙ ЗАДАЧИ ОПТИМАЛЬНОЙ ЭКСПЛУАТАЦИИ СИСТЕМЫ С БИНАРНОЙ СТРУКТУРОЙ ${ }^{1}$
}

\author{
Вл. Д. Мазуров, А. И. Смирнов
}

\begin{abstract}
Ранее авторами была доказана эквивалентность задачи устойчивой эксплуатации системы возобновляемых ресурсов некоторой задаче математического программирования. В данной статье изучаются свойства отображения, описывающего зависимость вектора состояния системы от управляющего воздействия. В частном случае структурированной популяции, описываемой бинарной моделью Лесли, охарактеризованы условия на целевую функцию, при которых существуют сохраняющие все структурные подразделения системы оптимальные управления. При этом используется обобщение классического понятия неразложимости отображения - понятие локальной неразложимости.

Ключевые слова: оптимальная эксплуатация популяций, сохраняющие управления, неразложимое отображение, вогнутое программирование.

Vl. D. Mazurov, A.I.Smirnov. A criterion for the existence of nondestructive controls in the problem of optimal exploitation of an ecosystem with a binary structure.

Earlier the authors proved the equivalence of a proposed formulation of a renewable ecoresource sustainable exploitation problem (based on representing the exploited ecosystem by a discrete dynamic system) and a certain mathematical program. In this paper we prove the concavity of a map describing the dependence of the state vector of the ecosystem on the control in the case where the step operator of the dynamic system is concave. In the particular case of a structured ecosystem described by Leslie's binary model, conditions for the objective function are characterized under which there are optimal controls preserving all structural divisions of the system. In this case, we used the notion of local irreducibility, which generalizes the classical notion of map irreducibility.

Keywords: rational exploitation of ecosystems, optimal nondestructive controls, irreducible map, concave programming.
\end{abstract}

MSC: $47 \mathrm{~N} 05,37 \mathrm{~N} 25,37 \mathrm{~N} 40$

DOI: $10.21538 / 0134-4889-2020-26-3-101-117$

\section{1. Введение. Постановка задачи и основные определения}

Одной из актуальных проблем, требующих привлечения математических методов, является задача рационального использования возобновляемых природных ресурсов. Наблюдаемая в последние десятилетия чрезмерная эксплуатация некоторых промысловых видов животных и растений приводит к непосильной нагрузке на регуляторные механизмы восстановления популяций и, как следствие, к их деградации и истощению.

Так, по данным ООН за 2018 г., продолжается сокращение площадей лесных массивов, которое является одним из ведущих факторов неблагоприятных изменений климата. Планета теряет до 200 тысяч гектаров леса ежегодно. Исследования показывают, что $40 \%$ лесных водосборов, поставляющих три четверти доступной пресной воды в мире, потеряли более половины своего первоначального древесного покрова. В период с 1990 по 2015 г. площади лесов сократились с 31,6\% до 30,6\% мировых земель. Россия относится к странам с крупнейшими лесопотерями. Согласно исследованиям Всемирного фонда природы (World Wildlife Fund), скорость утраты первозданных лесов из-за рубок и создания лесной инфраструктуры в России в 2016 - 2017 г.г. превышала отметку в 500 тыс. га ежегодно. Если в $2001-2002$ гг. от

\footnotetext{
${ }^{1}$ Исследования поддержаны грантом РФФИ (проект 19-07-01243).
} 
лесозаготовительной деятельности в нашей стране ежегодно терялось около 100 тыс. га самых экологически ценных, первозданных лесов, то в последние годы скорость их утраты выросла в 5 раз $[1 ; 2]$.

В рыболовстве оценить запасы биоресурсов намного сложнее; тем не менее существующие методики позволяют с высокой степенью надежности утверждать, что и здесь ситуация критическая - более $70 \%$ мировых рыбных запасов истощены; более 700 морских видов находятся под угрозой исчезновения [3].

Потребности определения обоснованных квот вылова именно в промысловом рыболовстве явились в середине прошлого века стимулом к разработке и использованию адекватных математических методов оценки состояния эксплуатируемых популяций и допустимых промысловых нагрузок. Пионерские работы здесь учитывали только общую биомассу популяции: использовалась т. н. модель избыточного производства Гордона - Шефера (другие названия модель биомассы или surplus-модель) [4].

Ранние работы [5;6], учитывающие структуру популяции, первоначально возрастную, затем и стадийную, использовали линейную постановку, при которой переход из предыдущего состояния популяции в следующее осуществляется матрицей, называемой проекиионной матрицей. Трудности, связанные с динамическими аспектами задачи, преодолевались рассмотрением только стационарных состояний. В качестве последних использовались собственные векторы, соответствующие доминирующему собственному значению проекционной матрицы.

В статье [7] понятие устойчивой эксплуатации было сформулировано для популяции с возрастной структурой - рассматривалась задача поиска максимального уровня допустимой эксплуатации, при котором определенная функция (доход от данного уровня эксплуатации или суммарная изымаемая биомасса) не убывает, начиная с некоторого момента времени, и доказана разрешимость этой задачи.

В [8] были формализованы некоторые естественные для задачи эксплуатации популяции понятия и формулировки, а также строго доказаны соответствующие утверждения. Например, тот факт, что допустима эксплуатация только имеющей достаточно высокий потенциал развития популяции, отражается в доказанном здесь утверждении о необходимости условия $\lambda(A)>1$ для возможности какого-либо регулярного изъятия особей из данной популяции.

Первые успешные попытки обобщений этих результатов на случай нелинейной зависимости от плотности связаны с работами $[9 ; 10]$, в которых изучалась зависимость от плотности только для первого возрастного класса. В [11] исследовалась модель, в которой зависимость от плотности распространена на все возрастные классы, а также на коэффициенты дожития и репродукции.

Подробный обзор различных подходов к постановке задачи оптимальной эксплуатации популяции, а также способов их решения содержится в монографии [12]. Заметим, что в подавляющем большинстве исследований по оптимальной эксплуатации на основе изъятия ресурсов из системы, даже если в первоначальной формулировке задачи используется аддитивное управление, как правило, в дальнейшем переходят к пропорциональному изъятию, т. е. к мультипликативному управлению, что упрощает нахождение и анализ оптимальных стратегий.

Приведенные выше исследования в качестве биологической основы использовали различные разновидности и обобщения модели Лесли возрастной структуры популяции. Проекционная матрица этой модели, называемая матрицей Лесли, имеет следующий вид:

$$
L=\left[\begin{array}{ccccc}
f_{1} & f_{2} & \ldots & f_{n-1} & f_{n} \\
s_{1} & 0 & \ldots & 0 & 0 \\
0 & s_{2} & \ldots & 0 & 0 \\
\vdots & \vdots & \ldots & \vdots & \vdots \\
0 & 0 & \ldots & s_{n-1} & 0
\end{array}\right]
$$

Здесь $f_{i}$ и $s_{i}$ - коэффициенты репродукции и дожития в соответствующих возрастных группах. Мы не будем здесь останавливаться на биологических предположениях, используемых 
при выводе этой модели; подробный обзор имеющихся моделей экологических популяций и их биологических аспектов содержится, например, в [13].

Дальнейшее развитие модели Лесли происходило в основном в двух направлениях. В рамках первого направления осуществлялся переход от возрастной структуры к более общей стадийной структуре; второе направление связано с учетом дополнительной (статусной) структуры, и, тем самым, с переходом к двумерной (бинарной) структуре популяции. Так, в одной из пионерских работ первого направления в качестве основания для классификации использовались последовательные стадии развития организма [14]. Альтернативные возрасту признаки классификации рассматривались в [5]; при моделировании популяций деревьев в лесоводстве учитывался их размер.

Позднее появился подход, когда учет стадийной структуры является не альтернативой, а дополнением к использованию возрастной структуры. Состояние популяции описывалось уже не вектором, а некоторой матрицей, имеющей блочную структуру. Исследование и построение таких моделей потребовало развития специального математического аппарата для работы с такими матрицами, использующего операцию Кронекера умножения матриц [15].

В рамках второго направления дополнительными признаками классификации, помимо возраста и размера, могут быть, например, такие характеристики, как объем, биомасса, генотип (фенотип), физиологические параметры особей, ареал обитания и т. д.

Так, в [16] особи классифицировались не только по возрасту, но и по количеству репродуктивных актов. Здесь впервые появилось обобщение матрицы Лесли - матрица той же структуры, имеющая вместо числовых элементов квадратные матрицы. Такого рода блочные матрицы в тех или иных вариациях присутствуют в работах поздних авторов моделей бинарной структуры популяций.

Отдельный класс моделей структуры популяций образуют т. н. мультирегиональные модели [17], описывающие систему нескольких взаимодействующих популяций с различными ареалами обитания. Состояние этих популяций определяется не только их демографическими параметрами, но и уровнем миграции между ними.

Обзор современного состояния моделирования динамики структурированных популяций содержится в [18].

Одна из нелинейных математических моделей, учитывающих двумерную структуру популяции, была создана под руководством д.ф.-м.н. Вл.Д. Мазурова для изучения вопроса о зависимости адаптационных возможностей популяции от ее генетической структуры. Данная модель описывала устойчивую динамику генетико-возрастной структуры одной конкретной природной популяции земноводных. Она основывалась на соответствующей экологической модели механизмов регуляции численности данной популяции. Позднее эта математическая модель была обобщена на некоторый достаточно общий класс дискретных динамических систем (эти результаты приведены в [19]). Именно это нелинейное обобщение модели Лесли будет использоваться нами далее в качестве экологической основы для задачи оптимальной эксплуатации популяции.

Настоящая статья является продолжением серии публикаций авторов по формализации понятия устойчивой эксплуатации экологической популяции. Согласно нашему подходу динамика состояний эксплуатируемой системы описывается итерационным процессом с оператором шага, принадлежащим некоторому достаточно общему классу отображений. Исследуются свойства полученной задачи оптимизации с аддитивными управлениями.

Необходимость разработки и исследования свойств общих моделей подчеркивается в монографии [4]: такого рода теоретические модели (называемые авторами компактными или глобальными) с изученными свойствами должны служить базой для создания более развернутых моделей конкретных природных систем. Основные свойства построенных подробных конкретных моделей вытекают из соответствующих свойств базовых общих моделей.

Исходная формализация задачи оптимальной эксплуатации в случае существования нетривиального равновесия дискретной динамической системы сведена в [20] к некоторой общей за- 
даче математического программирования. В [21] получена характеристика т. н. сохраняющих управлений - управлений, которые сохраняют все структурные подразделения популяции. Оказалось, что в случае вогнутого оператора шага динамической системы доминирующее собственное значение производной (в случае ее существования) для вектора состояния, соответствующего сохраняющему оптимальному управлению, должно равняться единице (в отличие от других допустимых и оптимальных управлений). В [22] получен критерий существования сохраняющих управлений на положительной границе допустимого множества для популяции с бинарной структурой, моделируемой обобщением модели Лесли.

Цель настоящей статьи - исследовать свойства зависимости равновесного вектора состояния дискретной динамической системы с вогнутым оператором шага от управления, а также получить условия существования оптимальных сохраняющих управлений задачи эксплуатации популяции с бинарной структурой, моделируемой нелинейным обобщением модели Лесли.

Далее будут использоваться следующие обозначения:

$\mathbb{R}_{+}^{q}$ - неотрицательный ортант пространства $\mathbb{R}^{q} ;$

$\mathbb{R}_{+}$- неотрицательная часть числовой прямой;

$x \leq y \Longleftrightarrow y-x \in \mathbb{R}_{+}^{q}, x \lessgtr y \Longleftrightarrow x \leq y, x \neq y$;

int $M-$ внутренность множества $M$;

со $M$ - выпуклая оболочка элементов множества $M$;

$\mathbb{Z}$ - множество целых чисел;

$\overline{m, n}=\{i \in \mathbb{Z} \mid m \leq i \leq n\}$

$\langle\cdot, \cdot\rangle-$ символ скалярного произведения.

Вектор $x=\left(x_{1}, x_{2}, \ldots, x_{q}\right)$ иногда кратко будем записывать в виде $x=\left(x_{i}\right)$. Итерации отображения $F$ определяются как $F^{t}=F\left(F^{t-1}\right)(t=1,2, \ldots), F^{0}(x) \equiv x$. Множество ненулевых (соответственно положительных) неподвижных точек отображения $F$ обозначается $N_{F}$ (соответственно $N_{F}^{+}$), максимальный элемент множества $N_{F}-\bar{x}_{F}$. Монотонно возрастающее отображение будем кратко называть монотонным, монотонно убывающее - антимонотонным.

Поскольку возможны иные содержательные интерпретации рассматриваемой задачи, будем использовать также нейтральный термин "система" для объекта эксплуатации.

\section{2. Некоторые предварительные результаты}

Эксплуатируемая система моделируется итерационным процессом

$$
x_{t+1}=F_{u}\left(x_{t}\right), \quad t=0,1,2, \ldots,
$$

где $F_{u}(x)=F(x)-u ; F \in\left\{\mathbb{R}_{+}^{q} \mapsto \mathbb{R}_{+}^{q}\right\}$; вектор $u$ таков, что $x_{t} \geq 0(t=0,1,2, \ldots)$. Компоненты вектора $x_{t}$ определяют численность (плотность, биомассу) структурных единиц системы, состоящей из $q>1$ подразделений, в момент времени $t(t=0,1,2, \ldots)$; компоненты вектора $u$ определяют объемы изъятий из соответствующих структурных подразделений.

Предполагается, что исходная система (в отсутствие управления) наряду с тривиальным равновесием $(F(0)=0$ ) имеет и нетривиальное равновесие (т. е. ее оператор шага $F$ имеет также ненулевую неподвижную точку).

В [20] была поставлена задача максимизации эффекта $c(u)$ эксплуатации системы на допустимом множестве $\bar{U}$, которое является замыканием множества $U$ сохраняющих управлений. Управляющий вектор $u$ называется сохраняющим, если хотя бы для одного начального состояния $x_{0}$ соответствующая траектория итерационного процесса (1) отделена от нуля, т. е. все подразделения системы стабильно существуют неограниченно долго.

Как мы увидим далее, множество сохраняющих управлений может быть открытым и тогда сохраняющие оптимальные управления отсутствуют. В этом случае оптимальными являются т. н. квазисохраняющие управления, которые элиминируют некоторые структурные подразделения системы (т. е. вектор состояния системы содержит нулевые координаты). Сохраняющие и квазисохраняющие управления поддерживают устойчивую динамику системы. 
Необходимым условием существования (квази)сохраняющих управлений является условие $N_{F}^{+} \neq \varnothing$ существования положительной неподвижной точки отображения $F$. Как и в предыдущих публикациях, будем предполагать отображение $F$ неотрицательным и вогнутым на $\mathbb{R}_{+}^{q}$. Поскольку такие отображения не только непрерывны на $\operatorname{int} \mathbb{R}_{+}^{q}$, но и имеют непрерывное расширение на $\mathbb{R}_{+}^{q}\left[23\right.$, теорема 10.3], будем считать отображение $F$ непрерывным на всем $\mathbb{R}_{+}^{q}$.

При необходимости будет привлекаться условие (локальной или глобальной) неразложимости отображения $F$. Неразложимость в классическом понимании [24] будем здесь называть глобальной неразложимостью. Определение и свойства локальной неразложимости отображения можно найти в статье авторов (Мазуров Вл. Д., Смирнов А. И. Условия неразложимости и примитивности монотонных субоднородных отображений // Тр. Ин-та математики и механики УрО РАН. 2016. Т. 22, № 3. С. 169-177).

Предполагается также выполненным следующее достаточное [21] для существования нетривиального равновесия исходной динамической системы (в отсутствие управления, т.е. при $u=0)$ условие

$$
\lambda\left(F_{\infty}\right)<1<\lambda\left(F_{0}\right)
$$

где $F_{0}(x)=\lim _{\alpha \rightarrow+0} \alpha^{-1} F(\alpha x), F_{\infty}(x)=\lim _{\alpha \rightarrow+\infty} \alpha^{-1} F(\alpha x)$.

Неразложимость в нуле отображения $F$ гарантирует положительность этого нетривиального равновесия. Следствием предположения (2) для вогнутого на $\mathbb{R}_{+}^{q}$ неразложимого в нуле отображения $F$ является ограниченность множества $N_{F}^{+}$, а также существование наибольшей неподвижной точки $\bar{x}_{F} \in N_{F}^{+}$этого отображения. В этих условиях задача оптимальной эксплуатации эквивалентна [20] задаче математического программирования вида

$$
\max \{c(u) \mid x=F(x)-u, x \geq 0, u \geq 0\},
$$

где $c(u)$ - неотрицательная монотонно возрастающая функция.

Обозначим через $\tilde{u}$ и $\widetilde{U}$ оптимальное множество и оптимальный вектор задачи (3); через $N_{u}$ и $N_{u}^{+}$- множества ненулевых и положительных неподвижных точек отображения $F_{u}$ соответственно. Допустимое множество $\bar{U}$ задачи (3) является замыканием множества $U$ сохраняющих управлений (для которых $x>0$ ). Эти множества представимы [20] в виде

$$
U=\left\{u \in \mathbb{R}_{+}^{q} \mid N_{u}^{+} \neq \varnothing\right\}, \quad \bar{U}=\left\{u \in \mathbb{R}_{+}^{q} \mid N_{u} \neq \varnothing\right\} .
$$

Из ограниченности множества $N_{F}^{+}$вытекает ограниченность множеств $\bar{U}$ и $N_{u}$ при $u \in \bar{U}$.

В указанных выше предположениях множество $U$ выпукло, содержит положительный вектор и вместе с каждым вектором $u$ - весь отрезок $[0, u][20]$.

При исследовании свойств задачи (3) в [21] было определено множество

$$
D=\left\{u \in \mathbb{R}_{+}^{q} \mid N_{u} \neq \varnothing, \quad N_{v}=\varnothing(\forall v>u)\right\},
$$

которое является частью границы множества сохраняющих управлений $U$ (см. представление (4)). Некоторые источники называют подобные подмножества границы, содержащие положительные точки вместе с предельными к последним (в относительной топологии), положительной границей. Поскольку целевая функция $c(u)$ является неотрицательной и монотонной, положительная граница $D$ содержит все потенциально оптимальные управления. Это множество может быть представлено в виде объединения двух непересекающиеся частей $D^{\prime}$ и $D^{\prime \prime}$, где

$$
D^{\prime}=\left\{u \mid N_{u}^{+} \neq \varnothing, N_{v}=\varnothing(\forall v>u)\right\}, \quad D^{\prime \prime}=\left\{u \in \mathbb{R}_{+}^{q} \mid N_{u} \neq \varnothing, N_{u}^{+}=\varnothing\right\} .
$$

Очевидно, $D^{\prime}=D \cap U, D^{\prime \prime}=D \backslash U$. Эти равенства означают, что оптимальное управление $\tilde{u}$ является сохраняющим тогда и только тогда, когда $\tilde{u} \in D^{\prime}$.

Непустое множество $N_{u}$ имеет наибольший элемент. Охарактеризуем его свойства. 
Утверждение 1. Пусть отображсние $F \in\left\{\mathbb{R}_{+}^{q} \mapsto \mathbb{R}_{+}^{q}\right\}$ является вогнутым и неразложимым в нуле, выполняется условие $(2)$ и $N_{u} \neq \varnothing$. Тогда множество $N_{u}$ содержит наибольший элемент $\bar{x}_{u}$, причем отображение $\bar{x}(u): u \rightarrow \bar{x}_{u}$ является вогнутым и монотонно (строго) убъвающим на $\bar{U}$ :

$$
N_{u} \neq \varnothing, \quad 0 \leq v \leq u \Rightarrow \bar{x}_{v} \geq \bar{x}_{u}, \quad 0 \leq v<u \Rightarrow \bar{x}_{v}>\bar{x}_{u} .
$$

Если к тому же отображение $F$ является глобально неразложимым, то отображение $\bar{x}(u)$ является сильно убывающим на $\bar{U}$ :

$$
0 \leq v \lessgtr u \Rightarrow \bar{x}_{v}>\bar{x}_{u}
$$

и глобалъно неразложимым. Кроме того, справедливы следующие заключения:

(1) если выполнено условие

$$
F(x)-F(y)=x-y, 0<x<y \Rightarrow F((1-\alpha) x+\alpha y)>(1-\alpha) F(x)+\alpha F(y)(\forall \alpha \in[0,1]),
$$

то отображение $\bar{x}(u)$ является непрерывным на всем $\bar{U}$;

(2) для любых $u^{(1)}, u^{(2)} \in \bar{U}$ справедливо неравенство

$$
\bar{x}\left(\alpha u^{(1)}+\beta u^{(2)}\right) \gtreqless \alpha \bar{x}\left(u^{(1)}\right)+\beta \bar{x}\left(u^{(2)}\right) \Rightarrow \bar{x}\left(\alpha u^{(1)}+\beta u^{(2)}\right)>\alpha \bar{x}\left(u^{(1)}\right)+\beta \bar{x}\left(u^{(2)}\right),
$$

где $\alpha>0, \beta>0, \alpha+\beta=1$. Таким образом, выполняется одно из двух условий:

$$
\begin{aligned}
& \bar{x}\left(\alpha u^{(1)}+\beta u^{(2)}\right)>\alpha \bar{x}\left(u^{(1)}\right)+\beta \bar{x}\left(u^{(2)}\right), \\
& \bar{x}\left(\alpha u^{(1)}+\beta u^{(2)}\right)=\alpha \bar{x}\left(u^{(1)}\right)+\beta \bar{x}\left(u^{(2)}\right) ;
\end{aligned}
$$

В последнем случае также справедливо равенство

$$
F\left(\alpha \bar{x}\left(u^{(1)}\right)+\beta \bar{x}\left(u^{(2)}\right)\right)=\alpha F\left(\bar{x}\left(u^{(1)}\right)\right)+\beta F\left(\bar{x}\left(u^{(2)}\right)\right) .
$$

Д о к а з а т е л ь с т в о. Покажем существование наибольшего элемента в множестве $N_{u}$. Для $x_{u} \in N_{u}$ справедливо $F\left(x_{u}\right)=x_{u}+u \geq x_{u}$, и, следовательно, последовательность $\left\{F^{t}\left(x_{u}\right)\right\}_{t=0}^{+\infty}$ монотонно возрастает. Из положительности неподвижной точки $\bar{x}_{F}$ отображения $F$ следует, что $x_{u} \leq \beta \bar{x}_{F}$ при некотором $\beta>1$. Но тогда $F^{t}\left(x_{u}\right) \leq F^{t}\left(\beta \bar{x}_{F}\right) \leq \beta F^{t}\left(\bar{x}_{F}\right)=$ $\beta \bar{x}_{F}$, так что эта последовательность является к тому же ограниченной и, следовательно, сходится к конечному пределу $x_{F} \in N_{F}$. Из неравенства $F\left(x_{u}\right) \geq x_{u}$ следует, что $x_{u} \leq x_{F} \leq \bar{x}_{F}$. Отсюда $F_{u}\left(\bar{x}_{F}\right) \geq F_{u}\left(x_{u}\right)=x_{u}$ и $\left(F_{u}\right)^{t}\left(x_{F}\right) \geq x_{u}(t=0,1,2, \ldots)$. С другой стороны, в силу $F_{u}\left(x_{F}\right) \leq F\left(x_{F}\right)=x_{F}$ последовательность $\left\{\left(F_{u}\right)^{t}\left(x_{F}\right)\right\}_{t=0}^{+\infty}$ является монотонно убывающей и потому сходится к конечному пределу. Из неравенств $\left(F_{u}\right)^{t}\left(x_{F}\right) \geq x_{u}\left(\forall x_{u} \in N_{u}, t=0,1,2, \ldots\right)$ следует, что ее предел $\bar{x}_{u} \in N_{u}$ - наибольший элемент множества $N_{u}$.

Докажем теперь монотонность отображения $\bar{x}(u)$. Пусть сначала $0 \leq v \leq u$. Тогда имеем $F_{v}\left(\bar{x}_{u}\right)=F\left(\bar{x}_{u}\right)-v=\bar{x}_{u}+(u-v) \geq \bar{x}_{u}$, т. е. $F_{v}\left(\bar{x}_{u}\right) \geq \bar{x}_{u}$ и последовательность $\left\{\left(F_{v}\right)^{t}\left(\bar{x}_{u}\right)\right\}_{t=0}^{+\infty}$ является монотонно возрастающей. В силу неравенств $F_{v}\left(\bar{x}_{u}\right) \leq F\left(\bar{x}_{u}\right) \leq F\left(\bar{x}_{F}\right)=\bar{x}_{F}$ она является к тому же ограниченной; поэтому имеет конечный предел $x_{v} \in N_{v}$. Из неравенства $F_{v}\left(\bar{x}_{u}\right) \geq \bar{x}_{u}$ получаем $x_{v} \geq \bar{x}_{u}$; тем более $\bar{x}_{v} \geq \bar{x}_{u}$.

Если же $0 \leq v<u$, то $F_{v}\left(\bar{x}_{u}\right)>\bar{x}_{u}$, и, проводя те же рассуждения, что и выше, получаем $x_{v}>\bar{x}_{u}$ и $\bar{x}_{v}>\bar{x}_{u}$.

Докажем вогнутость отображения $\bar{x}(u)$. Для $u^{(1)}, u^{(2)} \in \bar{U}$ введем в рассмотрение векторы

$$
u_{\alpha}=(1-\alpha) u^{(1)}+\alpha u^{(2)}, \quad x_{\alpha}=(1-\alpha) x^{(1)}+\alpha x^{(2)}(\alpha \in[0,1]), \quad x^{(1)}=\bar{x}_{u^{(1)}}, \quad x^{(2)}=\bar{x}_{u^{(2)}} .
$$

Используя вогнутость отображения $F$, для $F_{u_{\alpha}}\left(x_{\alpha}\right)=F\left(x_{\alpha}\right)-u_{\alpha}$ получаем

$$
F_{u_{\alpha}}\left(x_{\alpha}\right) \geq(1-\alpha) F\left(x^{(1)}\right)+\alpha F\left(x^{(2)}\right)-u_{\alpha}
$$




$$
=(1-\alpha) F_{u^{(1)}}\left(x^{(1)}\right)+\alpha F_{u^{(2)}}\left(x^{(2)}\right)=(1-\alpha) x^{(1)}+\alpha x^{(2)}=x_{\alpha},
$$

т. е. $F_{u_{\alpha}}\left(x_{\alpha}\right) \geq x_{\alpha}$. Поэтому реализация $\left\{\left(F_{u_{\alpha}}\right)^{t}\left(x_{\alpha}\right)\right\}_{t=0}^{+\infty}$ итерационного процесса (1) монотонно возрастает. Поскольку она также ограничена сверху (например, вектором $\bar{x}_{F}$ ), то является сходящейся. Для ее предела $\bar{x} \in N_{u}$ справедливо $\bar{x} \geq x_{\alpha}$. Таким образом, находим, что

$$
\bar{x}\left((1-\alpha) u^{(1)}+\alpha u^{(2)}\right) \geq \bar{x} \geq x_{\alpha}=(1-\alpha) x^{(1)}+\alpha x^{(2)}=(1-\alpha) \bar{x}_{u^{(1)}}+\alpha \bar{x}_{u^{(2)}},
$$

и тем самым вогнутость отображения $\bar{x}(u)$ доказана.

Пусть теперь отображение $F$ является глобально неразложимым. Докажем сильную антимонотонность отображения $\bar{x}(u)$.

Действительно, предположив противное, получаем для $u \supsetneqq v$ существование равных координат у векторов $\bar{x}(u), x(v): \quad \bar{x}_{i}(u)=\bar{x}_{i}(v)$ при некотором $i \in \overline{1, q}$. Но тогда из равенства $F_{v}\left(\bar{x}_{u}\right)=\bar{x}_{u}+(u-v)$ и доказанной выше монотонной сходимости последовательности $\left\{\left(F_{v}\right)^{t}\left(\bar{x}_{u}\right)\right\}_{t=0}^{+\infty}$ к $\bar{x}(v)$ вытекает, что $\bar{x}_{j}(v)>\bar{x}_{j}(u)$ для $u_{j}>v_{j}$. Поэтому $u_{i}=v_{i}$.

Далее, из равенств

$$
\bar{x}_{i}(u)=f_{i}(\bar{x}(u))-u_{i}, \quad \bar{x}_{i}(v)=f_{i}(\bar{x}(v))-v_{i}, \quad \bar{x}_{i}(u)=\bar{x}_{i}(v)
$$

имеем $f_{i}(\bar{x}(u))=f_{i}(\bar{x}(v))$, т. е. отображение $F$ разложимо в точке $\bar{x}(u)$. Полученное противоречие показывает, что $\bar{x}_{i}(u)<\bar{x}_{i}(v)(\forall i \in \overline{1, q})$, т. е. отображение $\bar{x}(u)$ является сильно убывающим.

Для доказательства глобальной неразложимости $\bar{x}(u)$ нам понадобится множество

$$
I^{0}(x, y)=\left\{i \in \overline{1, q} \mid x_{i}=y_{i}\right\}, \quad x=\left(x_{i}\right), \quad y=\left(y_{i}\right) .
$$

Пусть для некоторых векторов $v, w$ выполнены условия $v \gtreqless w, I^{0}(v, w) \neq \varnothing$. Поскольку отображение $\bar{x}(u)$ является сильно антимонотонным, из $v \gtreqless w$ следует, что $\bar{x}(v)<\bar{x}(w)$. Но это означает, что $I^{0}(\bar{x}(v), \bar{x}(w))=\varnothing$, так что условие разложимости $I^{0}(v, w) \subseteq I^{0}(\bar{x}(v), \bar{x}(w))$ не может быть выполнено. Глобальная неразложимость отображения $\bar{x}(u)$ доказана.

(1) Непрерывость отображения $\bar{x}(u)$ на $\operatorname{int} \bar{U}$ вытекает из его вогнутости; докажем его непрерывность на границе множества $\bar{U}$.

Сделаем это сначала для положительной границы $D=D^{\prime} \cup D^{\prime \prime}$ множества $\bar{U}$. Пусть $u^{k} \in \bar{U}(k=1,2, \ldots)$ - любая последовательность, сходящаяся к $u^{0} \in D$ при $k \rightarrow+\infty$. Покажем, что любая подпоследовательность последовательности $\left\{\bar{x}\left(u^{k}\right)\right\}_{k=1}^{+\infty}$ содержит подпоследовательность, сходящуюся к $x\left(u^{0}\right)$. Это будет означать сходимость самой последовательности к тому же пределу.

Сходящаяся подпоследовательность (пусть это будет $\left\{\bar{x}\left(u^{k_{\ell}}\right)\right\}_{\ell=1}^{+\infty}$ ) существует в силу ограниченности последовательности $\left\{\bar{x}\left(u^{k}\right)\right\}_{k=1}^{+\infty}$.

Поскольку $u^{k_{\ell}} \in \bar{U}$, то согласно $(4) \bar{x}\left(u^{k_{\ell}}\right) \in N_{u^{k_{\ell}}}$ и $\bar{x}\left(u^{k_{\ell}}\right)=F\left(\bar{x}\left(u^{k_{\ell}}\right)\right)-u^{k_{\ell}}(\ell=1,2, \ldots)$. Согласно нашим предположениям отображение $F$ непрерывно на $\bar{U}$, поэтому, переходя к пределу в последнем равенстве при $k \rightarrow+\infty$, получаем $\bar{x}=F(\bar{x})-u^{0}$, т. е. $\bar{x} \in N_{u^{0}}$.

Если $u \in D^{\prime \prime}$, то в силу единственности $\bar{x}\left(u^{0}\right)$ в множестве $N_{u^{0}}[21$, следствие 4] имеем $\bar{x}=\bar{x}\left(u^{0}\right)$, т. е. подпоследовательность $\left\{\bar{x}\left(u^{k_{\ell}}\right)\right\}_{\ell=1}^{+\infty}$ сходится к $\bar{x}\left(u^{0}\right)$.

Пусть теперь $u \in D^{\prime}$. Покажем, что и в этом случае $\bar{x}=\bar{x}\left(u^{0}\right)$. Действительно, предположив противное, в силу глобальной неразложимости отображения $F$ получаем неравенство $\bar{x}<\bar{x}\left(u^{0}\right)$, которое приводит к существованию в $N_{u^{0}}$ бесконечного числа элементов вида $\bar{x}_{\alpha}=(1-\alpha) \bar{x}+\alpha \bar{x}\left(u^{0}\right)(\alpha \in[0,1])$ [21, теорема 4]. Отсюда находим следующие равенства:

$$
\begin{gathered}
F\left(\bar{x}_{\alpha}\right)-u^{0}=(1-\alpha) \bar{x}+\alpha \bar{x}\left(u^{0}\right) \\
=(1-\alpha)\left(F(\bar{x})-u^{0}\right)+\alpha\left(F\left(\bar{x}\left(u^{0}\right)-u^{0}\right)=(1-\alpha) F(\bar{x})+\alpha F\left(\bar{x}\left(u^{0}\right)-u^{0},\right.\right. \\
F\left((1-\alpha) \bar{x}+\alpha \bar{x}\left(u^{0}\right)\right)=(1-\alpha) F(\bar{x})+\alpha F\left(\bar{x}\left(u^{0}\right) .\right.
\end{gathered}
$$


Наконец, из равенств $\bar{x}=F(\bar{x})-u^{0}, \bar{x}\left(u^{0}\right)=F\left(\bar{x}\left(u^{0}\right)\right)-u^{0}$ получаем $F\left(\bar{x}\left(u^{0}\right)\right)-F(\bar{x})=\bar{x}\left(u^{0}\right)-\bar{x}$. Но тогда равенство (12) противоречит условию (7). Таким образом, $\bar{x}=\bar{x}\left(u^{0}\right)$ и в этом случае, и непрерывность отображения $\bar{x}(u)$ на положительной границе $D$ доказана.

Непрерывность отображения $\bar{x}(u)$ на остальной, линейной части $\partial U \backslash D$ границы множества $\bar{U}$ (образованной координатными гиперплоскостями неотрицательного ортанта) вытекает [23, теорема 10.3] из существования симплициальной части множества $\bar{U}$, имеющей ту же линейную границу, что и $\bar{U}$. Действительно, если $v_{1}, v_{2}, \ldots, v_{q}$ - точки множества $\bar{U}$ на координатных осях, то в силу выпуклости множества $\bar{U}$ симплекс со $\left\{0, v_{1}, v_{2}, \ldots, v_{q}\right\}$ содержится в $\bar{U}$, и линейная часть $\partial U \backslash D$ границы $\bar{U}$ является также границей этого симплекса.

(2) Докажем справедливость равенств (8) или (9), (10).

Для векторов $y=\bar{x}\left(u_{\alpha}\right), y^{\prime}=x_{\alpha}$ (см. (11)) имеем $y \geq y^{\prime}$. Предположим, что $I^{0}=$ $I^{0}\left(y, y^{\prime}\right) \neq \varnothing$. Обозначим $a=(1-\alpha)\left(f_{i}\left(x^{(1)}\right)-u_{i}^{(1)}\right)+\alpha\left(f_{i}\left(x^{(2)}\right)-u_{i}^{(2)}\right)$. Учитывая монотонность отображения $F$ и равенства $\bar{x}\left(u^{(j)}\right)=F\left(\bar{x}\left(u^{(j)}\right)\right)-u^{(j)}(j=1,2)$, для всех $i \in I^{0}$ получаем

$$
\begin{gathered}
a=(1-\alpha) x_{i}^{(1)}+\alpha x_{i}^{(2)}=y_{i}^{\prime}=y_{i} \\
=f_{i}(y)-\left(u_{\alpha}\right)_{i} \geq f_{i}\left(y^{\prime}\right)-\left(u_{\alpha}\right)_{i} \geq(1-\alpha)\left(f_{i}\left(x^{(1)}\right)\right)+\alpha\left(f_{i}\left(x^{(2)}\right)\right)-\left(u_{\alpha}\right)_{i}=a .
\end{gathered}
$$

Отсюда получаем $f_{i}(y)=f_{i}\left(y^{\prime}\right)\left(\forall i \in I^{0}\right)$. При $I^{0} \neq \overline{1, q}$ это означает разложимость отображения $F$ в точке $y^{\prime}$, поэтому либо $I^{0}=\overline{1, q}$, и тогда справедливы равенства (9), (10), либо предположение $I^{0}\left(y, y^{\prime}\right) \neq \varnothing$ неверно, и тогда справедливо (8).

Все заключения утверждения доказаны.

Заметим, что условие (7), гарантирующее единственность неподвижной точки отображения $F_{u}$, является лишь достаточным для непрерывности отображения $\bar{x}(u)$ на $D^{\prime}$. Более сильным достаточным условием для единственности неподвижной точки отображения $F_{u}$ является строгая вогнутость отображения $F[21$, следствие 4].

Покажем существенность условия глобальной неразложимости в утверждении 1.

П р и м е р 1. Рассмотрим систему ограничений следующего вида:

$$
x_{1}=f\left(x_{2}\right)-u_{1}, \quad x_{2}=f\left(x_{2}\right)-u_{2}, \quad x_{1} \geq 0, \quad x_{2} \geq 0, \quad u_{1} \geq 0, \quad u_{2} \geq 0,
$$

где $f(a)=2 \min \{a, 1\}(a \geq 0)$. Данное отображение $F=\left(f_{1}(x), f_{2}(x)\right)$, где $f_{1}(x)=f\left(x_{2}\right)$, $f_{2}(x)=f\left(x_{1}\right)$, имеет единственную ненулевую неподвижную точку $\bar{x}_{F}=(2,2)$.

Очевидно, все предположения утверждения 1, кроме глобальной неразложимости, выполнены. Отображение $F$ здесь не является глобально неразложимым хотя бы потому, что для точек $\bar{x}_{F}=(2,2), \bar{x}=(2, t)$ при $t>2$ имеют место равенства $F\left(\bar{x}_{F}\right)=(2,2)=F(\bar{x})$, так что $\left(\bar{x}_{F}\right)_{1}=\bar{x}_{1}$ и $f_{1}\left(\bar{x}_{F}\right)=f_{1}(\bar{x})$.

Система ограничений (13) описывает часть неотрицательного ортанта, ограниченную частями прямых $u_{1}+2 u_{2}=3\left(u_{1} \in[0,1]\right)$ и $2 u_{1}+u_{2}=3\left(u_{1} \in[1,3 / 2]\right)$, которые и составляют множество $D^{\prime}$; множество $D^{\prime \prime}=\varnothing$. Для точек $u=(1,1), v=(1,0)$ имеем $v \varsubsetneqq u$, но $\bar{x}_{v}=(1,2) \ngtr \bar{x}_{u}=(1,1)$, т. е. отображение $\bar{x}(u)$ не является сильно убывающим.

Далее, для точек $u^{(1)}=(0,0), u^{(2)}=(3 / 2,0)$ имеем: $\bar{x}\left(u^{(1)}\right)=(2,2), x\left(u^{(2)}\right)=(1 / 2,1)$. При $\alpha=1 / 3, \beta=2 / 3$ находим $\bar{x}\left(\alpha u^{(1)}+\beta u^{(2)}\right)=\bar{x}(1 / 2,0)=(3 / 2,2), \alpha \bar{x}\left(u^{(1)}\right)+\beta \bar{x}\left(u^{(2)}\right)=(3 / 2,5 / 3)$, так что ни одно из равенств (8), (9) не выполнено.

Любой оптимальный вектор задачи (3) является либо сохраняющим, либо квазисохраняющим, в зависимости от вида целевой функции и свойств множеств $D^{\prime}, D^{\prime \prime}$; в частности, от того, какое из этих множеств является замкнутым. Множество $D^{\prime}$ может не быть ни замкнутым, ни открытым (см. пример в статье [22]). Покажем, что множество квазисохраняющих управлений $D^{\prime \prime}$ является замкнутым (в относительной топологии).

Утверждение 2. Пусть отображсение $F \in\left\{\mathbb{R}_{+}^{q} \mapsto \mathbb{R}_{+}^{q}\right\}$ является вогнутым, глобально неразложимым и выполняется условие (2). Тогда множество $D^{\prime \prime}$ квазисохраняющих управлений является замкнутым. 
Д о к аз а т ел ь с т в о. Пусть $u^{k} \rightarrow u^{0}$ при $k \rightarrow+\infty$, где $u^{k} \in D^{\prime \prime}(k=1,2, \ldots)$. Покажем, что $u^{0} \in D^{\prime \prime}$.

Из представления (6) множества $D^{\prime \prime}$ следует, что $N_{u^{k}} \neq \varnothing$ и имеют место равенства $\bar{x}\left(u^{k}\right)=F\left(\bar{x}\left(u^{k}\right)\right)-u^{k}(k=1,2, \ldots)$. Согласно заключению (1) утверждения 1 отображение $\bar{x}(u)$ является непрерывным на $\bar{U}$, причем, как видно из доказательства, свойство $(7)$ для непрерывности отображения $\bar{x}(u)$ на множестве $D^{\prime \prime}$ не требуется. Отсюда получаем сходимость $\bar{x}\left(u^{k}\right) \rightarrow \bar{x}\left(u^{0}\right)$ при $k \rightarrow+\infty$. Наконец, из равенств $\bar{x}\left(u^{k}\right)=F\left(\bar{x}\left(u^{k}\right)\right)-u^{k}(k=1,2, \ldots)$ получаем равенство $\bar{x}\left(u^{0}\right)=F\left(\bar{x}\left(u^{0}\right)\right)-u^{0}$, означающее, что $N_{u^{0}} \neq \varnothing$.

Далее, поскольку $u^{k} \in D^{\prime \prime}$, то согласно (6) $N_{u^{k}}^{+}=\varnothing(\forall k=1,2, \ldots)$ и все элементы последовательности $\left\{\bar{x}\left(u^{k}\right)\right\}_{k=1}^{+\infty}$ имеют нулевую координату; поэтому существует ее подпоследовательность, элементы которой имеют нулевые координаты на одном и том же месте: $\bar{x}_{i}\left(u_{k_{\ell}}\right)=0$ $(\forall \ell=1,2, \ldots)$. Переходя в этих равенствах к пределу при $\ell \rightarrow+\infty$, получаем $\bar{x}_{i}\left(u^{0}\right)=0$. Но это означает в силу (6), что $u^{0} \in D^{\prime \prime}$.

Доказательство завершено.

\section{3. Свойства сохраняющих и квазисохраняющих управлений для бинарной модели Лесли}

Обобщение модели Лесли, которое мы будем использовать далее, описывает популяцию с бинарной структурой: имеются $m$ ее подразделений, каждое из которых, в свою очередь, содержит особи $n$ возрастов (стадий). Если обозначить через $x_{i, j}^{(t)}$ число особей подразделения $i \in \overline{1, m}$ возраста $j \in \overline{1, n}$ в момент времени $t=0,1,2 \ldots$, то соотношения этой модели примут следующий вид:

$$
x_{i, 1}^{(t+1)}=f_{i}\left(a_{t}\right), a_{t}=\sum_{i=1}^{m} \sum_{j=1}^{n} \beta_{i, j} x_{i, j}^{(t)}, x_{i, j+1}^{(t+1)}=\alpha_{i, j} x_{i, j}^{(t)}(i \in \overline{1, m}, j \in \overline{1, n-1}, t=0,1,2, \ldots) .
$$

Здесь $\alpha_{i, j}>0$ и $\beta_{i, j} \geq 0-$ коэффициенты дожития и репродукции соответственно; $a_{t}-$ суммарное число новорожденных популяции в момент времени $t=0,1,2 \ldots$ Оператор шага динамической системы $(14)$ имеет вид $F(x)=\left(f_{i, j}(x)\right)$, где

$$
f_{i, 1}(x)=f_{i}(a(x)), \quad f_{i, j+1}(x)=\alpha_{i, j} x_{i, j} \quad(i \in \overline{1, m}, j \in \overline{1, n-1}) .
$$

Равновесное состояние системы (14) задается следующими равенствами:

$$
x_{i, j}=\pi_{j}^{(i)} f_{i}(a(x)), \quad \pi_{j}^{(i)}=\prod_{\ell=1}^{j-1} \alpha_{i, \ell} \quad(i \in \overline{1, m}, j \in \overline{1, n}), \quad a(x)=\sum_{i=1}^{m} \sum_{j=1}^{n} \beta_{i, j} x_{i, j} .
$$

Здесь вектор $x=\left(x_{i, j}\right)$ имеет блочную структуру: $x=\left(x^{(1)}, x^{(2)}, \ldots, x^{(m)}\right), x^{(i)}=\left(x_{i, 1}, \ldots, x_{i, n}\right)$ $(i \in \overline{1, m})$. Аналогичную структуру имеет вектор $F(x)=\left(f_{i, j}(x)\right)$ из $(15)$.

В матричных моделях популяций определяющую роль для асимптотического поведения дискретной динамической системы играет т. н. индикатор потенциального роста. Для модели (14) имеется его нелинейный аналог, определяемый следующим образом:

$$
\sigma(a)=\sum_{i=1}^{m} \sigma^{(i)} f_{i}(a), \quad \sigma^{(i)}=\sigma_{n}^{(i)}, \quad \sigma_{j}^{(i)}=\sum_{k=1}^{j} \beta_{i, k} \pi_{k}^{(i)} \quad(i \in \overline{1, m}, j \in \overline{0, n}) .
$$

Как мы увидим далее, величина $\sigma(a)$ является агрегированной характеристикой адаптационных возможностей моделируемой соотношениями (14) популяции, выражающихся, в частности, в ее способности иметь нетривиальное устойчивое равновесное состояние. 
Предполагается, что функции $f_{i}(a)$ удовлетворяют следующим условиям:

$$
f_{i}(0)=0, \quad f_{i}(a) \text { вогнуты на } \mathbb{R}_{+} \quad(i \in \overline{1, m}) .
$$

Следовательно, эти функции являются также монотонно возрастающими и положительными на int $\mathbb{R}_{+}$. Это означает, что нетривиальное равновесие $\bar{x}_{F}$ из (16) является положительным (даже в отсутствие требования неразложимости отображения (15) в нуле).

Предположение (2) о существовании положительного равновесия трансформируется в условие

$$
\sigma^{\prime}(+\infty)<1<\sigma^{\prime}(+0)
$$

Неравенства (18) означают существование положительного решения $\bar{a}_{F}$ уравнения $\sigma(a)=a$, которое связано с положительной неподвижной точкой $\bar{x}_{F}$ отображения (19) соотношением $\bar{a}_{F}=a\left(\bar{x}_{F}\right)$, где $a(x)$ определено в (16).

Неразложимость в нуле отображения (15) равносильна требованию

$$
\beta_{i, n}>0 \quad(\forall i \in \overline{1, m}) .
$$

Глобальная неразложимость отображения (15) равносильна неразложимости лишь в нуле при выполнении следующего усиления условия (19):

$$
\text { функции } f_{i}(a) \text { строго возрастают при } a \in[0,+\infty)(\forall i \in \overline{1, m}) \text {. }
$$

Введем следующие обозначения (здесь всюду $i \in \overline{1, m}, j, k \in \overline{1, n}$ ):

$$
\begin{gathered}
\pi^{(i)}=\pi_{n}^{(i)}, \quad \pi_{j}^{(i)}=p_{1, j}^{(i)}, \quad p_{j}^{(i)}=p_{j, n}^{(i)}, \quad p_{j, k}^{(i)}=\prod_{\ell=j}^{k-1} \alpha_{i, \ell}, \\
p^{(i)}(u)=p_{n}^{(i)}(u), \quad p_{j}^{(i)}(u)=\sum_{k=1}^{j} p_{k, j}^{(i)} u_{i, k}, \\
q(u)=\langle q, u\rangle, q=\left(q^{1}, q^{2}, \ldots, q^{m}\right), q^{i}=\left(q_{1}^{(i)}, q_{2}^{(i)}, \ldots, q_{n}^{(i)}\right), q_{j}^{(i)}=q_{j, n}^{(i)}, q_{j, k}^{(i)}=\sum_{s=j}^{k} \beta_{i, s} \prod_{t=j}^{s-1} \alpha_{i, t}, \\
\mu(a)=\sigma(a)-a, \quad \mu^{*}=\max _{a \geq 0} \mu(a), \quad \lambda_{i}(a)=\pi^{(i)} f_{i}(a), \quad \lambda_{i}^{*}=\lambda_{i}\left(a^{*}\right) .
\end{gathered}
$$

Допустимое множество $\bar{U}$ задачи (3) для модели (14) задается ограничениями

$$
x_{i, 1}=f_{i}(a(x))-u_{i, 1}, a(x)=\sum_{i=1}^{m} \sum_{j=1}^{n} \beta_{i, j} x_{i, j}, x_{i, j+1}=\alpha_{i, j} x_{i, j}-u_{i, j+1} \quad(i \in \overline{1, m}, j \in \overline{1, n-1}),
$$

где векторы $x, u$ неотрицательны. Найдем явные выражения для координат блоков $x^{(i)}$ :

$$
x_{i, j}=\pi_{j}^{(i)} f_{i}(a)-p_{j}^{(i)}(u)(j \in \overline{1, n-1}), x_{i, n}=\lambda_{i}(a)-p^{(i)}(u), a=\sum_{i=1}^{m} \sum_{j=1}^{n} \beta_{i, j} x_{i, j}(i \in \overline{1, m}) .
$$

Для вектора $\bar{x}(u)=\left(\bar{x}_{i, j}(u)\right)$ определим множество структурных подразделений, последние возрастные группы которых полностью элиминируются управлением $u$ :

$$
I_{0}(u)=\left\{i \in \overline{1, m} \mid \bar{x}_{i, n}(u)=0\right\} .
$$

Из (25) с учетом обозначений (21)-(24) получаем представления множеств (6):

$$
D^{\prime}=\left\{u \mid p^{(i)}(u)<\lambda_{i}^{*}(\forall i \in \overline{1, m}), \quad q(u)=\mu^{*}, \quad u \geq 0\right\}
$$




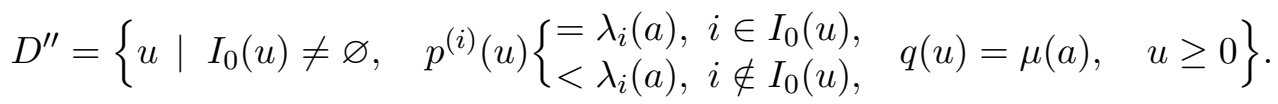

Множество $D^{\prime}$ принадлежит [22, теорема 1] гиперплоскости

$$
\Gamma=\left\{u \in \mathbb{R}^{m n} \mid q(u)=\mu^{*}\right\} .
$$

Более того, справедливы свойства

$$
D^{\prime}=\Gamma \cap U, \quad D^{\prime \prime} \cap \Gamma \subset \overline{D^{\prime}}, \quad \overline{D^{\prime}}=\Gamma \cap D .
$$

Последние равенства хорошо интерпретируются утверждением 2: точки границы между множествами $D^{\prime}$ и $D^{\prime \prime}$ принадлежат последнему, хотя являются предельными для обоих множеств.

Далее будут фигурировать упорядоченные наборы элементов множеств $\overline{1, m}, \overline{0, n}$, для обозначения которых естественно использовать символ вектор-строки: запись $I=\left(i_{1}, \ldots, i_{\ell}\right)$ означает, что имеется набор $I=\left\{i_{1}, \ldots, i_{\ell}\right\}$ с фиксированным порядком элементов $i_{1}, \ldots, i_{\ell}$.

Пусть $I=\left(i_{1}, \ldots, i_{\ell}\right) \subseteq \overline{1, m}, J=\left(j_{1}, \ldots, j_{\ell}\right) \subseteq \overline{0, n}$, причем второй из этих наборов допускает их повторения в отличие от первого: $|I|=\ell,|J| \in \overline{1, \ell}$. Обозначим

$$
\bar{S}_{j}^{(i)}(a)=\sigma_{j}^{i} f_{i}(a)+\sum_{k \neq i} \sigma^{k} f_{k}(a) \quad(i \in \overline{1, m}, j \in \overline{0, n}) .
$$

Множество $D^{\prime \prime} \neq \varnothing$ тогда и только тогда, когда $J^{*} \neq \varnothing[25$, теорема 1$]$, где

$$
\begin{gathered}
J^{*}=\left\{k \in \overline{1, m} \mid J_{k}^{*} \neq \varnothing\right\}, \quad J_{k}^{*}=\overline{1, n} \backslash I_{k}^{*} \quad(k \in \overline{1, m}), \\
I^{*}=\left\{k \in \overline{1, m} \mid I_{k}^{*} \neq \varnothing\right\}, \quad I_{k}^{*}=\left\{j \in \overline{1, n} \mid \bar{S}_{j-1}^{(k)}\left(a^{*}\right)<a^{*}\right\} \quad(k \in \overline{1, m}) .
\end{gathered}
$$

В силу монотонности $\bar{S}_{j}^{(i)}(a)$ по нижнему индексу имеем

$$
I_{k}^{*}=\overline{1, i_{k}^{*}}, \quad J_{k}^{*}=\overline{i_{k}^{*}+1, n} \quad\left(i_{k}^{*} \in \overline{0, n}, k \in \overline{1, m}\right), \quad \beta_{k, i_{k}^{*}}>0 \quad(\forall k \in \overline{1, m}) .
$$

Пусть $a_{j}^{(i)}-$ решение уравнения

$$
\bar{S}_{j-1}^{(i)}(a)=a \quad\left(a \geq a^{*}\right)
$$

при $j \in J_{i}^{*}$, когда оно разрешимо [25, лемма 1]. Управления $u(i, j)$ с единственной положительной координатой $u_{i, j}$ распределяются по множествам $D^{\prime}, D^{\prime \prime}$ следующим образом $[25$, лемма 2]:

$$
u(i, j) \in\left\{\begin{array}{ll}
D^{\prime}, & i \in I^{*}, j \in I_{i}^{*}, \\
D^{\prime \prime}, & i \in J^{*}, j \in J_{i}^{*},
\end{array} \quad u_{i, j}= \begin{cases}\mu^{*}\left(q_{j, n}^{(i)}\right)^{-1}, & i \in I^{*}, j \in I_{i}^{*}, \\
\pi_{j}^{i} f_{i}\left(a_{j}^{(i)}\right), & i \in J^{*}, j \in J_{i}^{*} .\end{cases}\right.
$$

Заметим, что равенства (31) корректны, поскольку $q_{j, n}^{(i)} \neq 0$ при $i \in I^{*}, j \in I_{i}^{*}$ в силу (23), (30). Используя обозначения (16), определим функцию

$$
\bar{a}(u)=a(\bar{x}(u)) \quad(u \in \bar{U}) .
$$

Согласно утверждению 1 эта функция является монотонно убывающей и вогнутой на $\bar{U}(a)$. Пусть $W-$ некоторое подмножество допустимого множества $\bar{U}$ и $\bar{a}_{W}=\sup \{\bar{a}(u) \mid u \in W\}$. Тогда, очевидно, $\bar{a}_{\bar{U}}=\bar{a}_{F}, \bar{a}_{D^{\prime}}=a^{*}$ и $\bar{a}_{D}=a_{D^{\prime \prime}}$ при $D^{\prime \prime} \neq \varnothing[25$, лемма 3]).

Допустимое множество $\bar{U}$ представимо в виде $\bar{U}=\cup\left\{\bar{U}(a) \mid a \in\left[0, \bar{a}_{F}\right]\right\}$, где

$$
\bar{U}(a)=\left\{u \mid p^{(i)}(u) \leq \lambda_{i}(a) \quad(\forall i \in \overline{1, m}), \quad q(u)=\mu(a), \quad u \geq 0\right\} .
$$


Пусть $D(a)=\bar{U}(a) \cap D^{\prime \prime}, V(a)$ - множество вершин многогранника $\bar{U}(a)$,

$$
V_{D^{\prime}}=V\left(a^{*}\right) \cap D^{\prime}, \quad V_{D^{\prime \prime}}(a)=V(a) \cap D^{\prime \prime} \quad\left(a \in\left[a^{*}, \bar{a}_{D^{\prime \prime}}\right]\right) .
$$

При доказательстве замкнутости множества $D^{\prime \prime}$ (утверждение 2) привлекалось предположение о глобальной неразложимости отображения $F$. Как показывает следующее утверждение, в частном случае отображения вида (15) оказывается возможным доказать замкнутость множества $D^{\prime \prime}$, не используя предположение о глобальной неразложимости отображения $F$. Здесь же дается представление множества $D^{\prime}$ в виде выпуклой оболочки множества точек из множества $D$ (или только из $D^{\prime \prime}$, если множество $I^{*}$ из (29) является пустым).

Утверждение 3. Пусть выполнены условия (17), (18). Тогда:

(1) множество $D^{\prime \prime}$ является замкнутым (в относительной топологии),

(2) множество $D^{\prime}$ является выпуклым, причем справедливо представление

$$
D^{\prime}=\left(\operatorname{co} V^{*}\right) \backslash D\left(a^{*}\right),
$$

где $V^{*}=V_{D^{\prime}} \cup V_{D^{\prime \prime}}, V_{D^{\prime}}=\left\{u(i, j) \mid i \in I^{*}, j \in I_{i}^{*}\right\}$.

Если $I^{*}=\varnothing$, то всякий $v \in D^{\prime}$ представи́м в виде

$$
v=\sum_{j=1}^{k} \alpha_{j} v_{j}, \quad \sum_{j=1}^{k} \alpha_{j}=1, \quad \alpha_{j}>0 \quad(\forall j \in \overline{1, k}),
$$

где $V=\left\{v_{1}, \ldots v_{k}\right\} \subseteq V\left(a^{*}\right)$, причем $V \not \subset L_{i}\left(a^{*}\right)(\forall i \in \overline{1, m})$.

Д ок а з а т ел ь с т в о.

(1) Пусть $u_{k} \rightarrow u_{0}$ при $k \rightarrow+\infty$, где $u_{k} \in D^{\prime \prime}$. Покажем, что $u_{0} \in D^{\prime \prime}$.

В силу представления $(27)$ имеем $q\left(u_{k}\right)=\mu\left(\bar{a}\left(u_{k}\right)\right)$, где функция $\bar{a}(u)$, которая выше определялась как $\bar{a}(u)=a(\bar{x}(u))$, может быть также определена как решение уравнения $q(u)=\mu(a)$. Действительно, функция $\mu(a)$ (см. определение (24)) непрерывна и строго убывает при $a \geq a^{*}[22$, лемма 1]), следовательно, имеет непрерывную и строго убывающую обратную $\mu^{-1}(\alpha)$ при $\alpha \in\left[\mu\left(\bar{a}_{D^{\prime \prime}}\right), \mu^{*}\right]$. Поэтому из равенств $q\left(u_{k}\right)=\mu\left(\bar{a}\left(u_{k}\right)\right), q\left(u_{0}\right)=\mu\left(\bar{a}\left(u_{0}\right)\right)$ при $k \rightarrow+\infty$ выводим $\bar{a}\left(u_{k}\right)=\mu^{-1}\left(q\left(u_{k}\right)\right) \rightarrow \mu^{-1}\left(q\left(u_{0}\right)\right)=\bar{a}\left(u_{0}\right)$, т. е. $\bar{a}\left(u_{k}\right) \rightarrow \bar{a}\left(u_{0}\right)$. Отсюда $\mathrm{c}$ учетом (22), (24) получаем $\lambda_{i}\left(\bar{a}\left(u_{k}\right)\right) \rightarrow \lambda_{i}\left(\bar{a}\left(u_{0}\right)\right), p^{(i)}\left(u_{k}\right) \rightarrow p^{(i)}\left(u^{0}\right)$ при $k \rightarrow+\infty(\forall i \in \overline{1, m})$.

Далее, из $(27)$ в силу $u_{k} \in D^{\prime \prime}$ имеем

$$
0=\bar{x}_{i, n}\left(u_{k}\right)=\lambda_{i}\left(\bar{a}\left(u_{k}\right)\right)-p^{(i)}\left(u_{k}\right) \rightarrow \lambda_{i}\left(\bar{a}\left(u_{0}\right)\right)-p^{(i)}\left(u^{0}\right) \quad(\forall i \in \overline{1, m}) .
$$

Отсюда $\bar{x}_{i, n}\left(u_{0}\right)=\lambda_{i}\left(\bar{a}\left(u_{0}\right)\right)-p^{(i)}\left(u_{0}\right)=0$. Поскольку остальные ограничения на $u_{0}, \bar{x}\left(u_{0}\right)$ в представлении (27) выполнены, получаем требуемое включение $u_{0} \in D^{\prime \prime}$.

(2) Выпуклость множества $D^{\prime}$ вытекает из линейности функций $q(u)$ и $p^{(i)}(u)(i \in \overline{1, m})$ в (26). Докажем представление $D^{\prime}$ в виде (34).

При фиксированном $a=a^{*}$ множество $\bar{U}\left(a^{*}\right)$ задается линейными ограничениями из (32) и, следовательно, является выпуклой коммбинацией его вершин $V\left(a^{*}\right)$. Из (32) также видно, что $\bar{U}\left(a^{*}\right) \subseteq \Gamma$ (см. (28)); согласно (26), (27), (32) имеем $\bar{U}\left(a^{*}\right)=D^{\prime} \cup D\left(a^{*}\right)$, причем $D^{\prime} \cap D\left(a^{*}\right)=\varnothing$ в силу $D\left(a^{*}\right) \subseteq D^{\prime \prime}$. Поскольку $V_{D^{\prime}}=\left\{u(i, j) \mid i \in I^{*}, j \in I_{i}^{*}\right\}$ [25, лемма 4, теорема 2], отсюда получаем для $D^{\prime}$ представление (34).

Если $I^{*}=\varnothing$, то $D^{\prime}=\left(\right.$ со $\left.V_{D^{\prime \prime}}\right) \backslash D\left(a^{*}\right)$, и справедливо представление $(35)$. Если все $v_{j}$ в этом представлении принадлежат некоторому (одному и тому же) множеству $L_{i}\left(a^{*}\right)(i \in \overline{1, m})$, то, учитывая включения $v_{j} \in D^{\prime \prime}(j \in \overline{1, k})$, в силу равенств $(25),(27)$ получаем

$\bar{x}_{i, n}(v)=\bar{x}_{i, n}\left(\sum_{j=1}^{k} \alpha_{j} v_{j}\right)=\lambda_{i}\left(a^{*}\right)-p^{(i)}\left(\sum_{j=1}^{k} \alpha_{j} v_{j}\right)=\sum_{j=1}^{k} \alpha_{j}\left(\lambda\left(a^{*}\right)-p^{(i)}\left(v_{j}\right)\right)=\sum_{j=1}^{k} \alpha_{j} \bar{x}_{i, n}\left(v_{j}\right)=0$.

Но это означает $v \in D^{\prime \prime}$, что противоречит условию $v \in D^{\prime}$. Поэтому $V \not \subset L_{i}\left(a^{*}\right) \quad(\forall j \in \overline{1, m})$.

Утверждение доказано. 


\section{4. Критерий существования сохраняющего оптимального управления}

Будем по-прежнему считать выполненными условия (17), (19), гарантирующие разрешимость задачи (3) для отображения (15). Представление (5) показывает, что оптимальный вектор $\tilde{u}$ должен принадлежать множеству $D=D^{\prime} \cup D^{\prime \prime}$, где множества $D^{\prime}$ сохраняющих и $D^{\prime \prime}$ квазисохраняющих управлений имеют представления (26), (27) соответственно. Прежде всего, естественно, нас будет интересовать вопрос о существовании сохраняющих оптимальных управлений $\tilde{u}$, для которых вектор состояния системы $\tilde{x}=\bar{x}(\tilde{u})$ положителен.

Обозначим

$$
\begin{gathered}
Q_{i}^{+}=\left\{j \in \overline{1, n} \mid q_{j, n}^{i}>0\right\}, \quad Q_{i}^{0}=\overline{1, n} \backslash Q_{+}^{i} \quad(i \in \overline{1, m}), \\
\gamma_{j}^{(i)}=c_{i, j}\left(q_{j, n}^{(i)}\right)^{-1} \quad\left(i \in \overline{1, m}, j \in Q_{i}^{+}\right), \\
\gamma=\max \left\{\gamma_{j}^{(i)} \mid i \in \overline{1, m}, j \in Q_{i}^{+}\right\} .
\end{gathered}
$$

Очевидно, $j \in Q_{i}^{+}$тогда и только тогда, когда $\sum_{k=j}^{n} \beta_{i, k}>0$. В частности, это неравенство справедливо при выполнении условия (19) неразложимости в нуле, когда $Q_{i}^{0}=\varnothing(\forall i \in \overline{1, m})$.

Утверждение 4. Пусть выполнены условия (17), (18). Сохраняющее оптимальное управление существует тогда и только тогда, когда выполнены следующие условия:

$$
\begin{gathered}
Q_{i}^{0} \subseteq C_{i}^{0} \quad(\forall i \in \overline{1, m}), \\
I^{*}=\varnothing \Rightarrow \gamma_{j}^{(i)}=\gamma \quad\left(\forall i \in \overline{1, m}, j \in Q_{i}^{+}\right), \\
I^{*} \neq \varnothing \Rightarrow \exists i_{0} \in I^{*}, j_{0} \in I_{i_{0}}^{*}: \quad \gamma=\gamma_{j_{0}}^{i_{0}} .
\end{gathered}
$$

Д о к а з а т е л ь с т в о. Пусть $I^{*}=\varnothing$. Тогда справедливо заключение (35) утверждения 3. Векторы $v_{j} \notin D^{\prime}(\forall j \in \overline{1, k})$, так что вектор $\tilde{u}$ из $D^{\prime}$ может быть оптимальным только в случае ортогональности вектора $c$ гиперплоскости $\Gamma$. Согласно обозначениям (23) это означает справедливость равенств $\left.c_{i, j} / q_{j, n}^{(i)}\right)=c_{i, k} / q_{k, n}^{(i)}(\forall i \in \overline{1, m}, j, k \in \overline{1, n})$, эквивалентных равенствам (37).

Пусть теперь сохраняющее оптимальное управление существует при $I^{*} \neq \varnothing$. Если $q_{j, n}^{(i)}=0$, то единичный орт $e_{i, j}$ параллелен гиперплоскости $\Gamma$ (см. (23)) и, следовательно, последняя вместе с каждой точкой $\tilde{u} \in \widetilde{U}$ содержит вектор $\tilde{u}(\alpha)=\tilde{u}+\alpha e_{i, j}(\forall \alpha \in[0,+\infty))$, причем $c(\alpha)=\langle c, \tilde{u}(\alpha)\rangle=\langle c, \tilde{u}\rangle+\alpha c_{i, j}$ неограниченно возрастает с ростом $\alpha$ при $c_{i, j} \neq 0$. Поэтому $c_{i, j}=0$ и, следовательно, выполнено (36).

Для доказательства справедливости (38) достаточно заметить, что в силу (33), (34) из $\widetilde{U} \cap D^{\prime} \neq \varnothing$ следует $\widetilde{U} \cap V_{D^{\prime}} \neq \varnothing$, т. е. среди векторов $u(i, j)$ с единственной ненулевой координатой из (31) имеется оптимальный. Равенство (38) является выражением того факта, что значение целевой функции для него является наибольшим.

Пусть теперь, наоборот, выполнены условия (36), (38). Поскольку из $\mu^{*}=\max _{a \geq 0} \mu(a)$ следует $q(u)=\mu(a) \leq \mu^{*}$, для любого $u \in D^{\prime \prime}$ согласно (27) найдется вектор $v \geq u, v \in \Gamma$ (если $u \in D^{\prime \prime} \cap \Gamma$, то $v=u$; в противном случае вектор $v$ уже не является допустимым), так что $\langle c, v\rangle \geq\langle c, u\rangle$. Условие (38) означает, что существует вектор $u(i, j)$, где $j \in I_{i}^{*}$, для которого $\langle c, u(i, j)\rangle \geq\langle c, v\rangle \geq\langle c, u\rangle$, т. е. $\langle c, u(i, j)\rangle \geq\langle c, u\rangle\left(\forall u \in D^{\prime \prime}\right)$. Ясно также, что $\langle c, u(i, j)\rangle \geq\langle c, w\rangle$ $\left(\forall w \in D^{\prime}\right)$. Таким образом, вектор $\tilde{u}=u(i, j) \in D^{\prime}$ является оптимальным.

Утверждение доказано.

П р и м е р 2. Будем использовать следующее допустимое множество, рассмотренное в [22]:

$$
x_{1}=f(a)-u_{1}, \quad x_{2}=\frac{1}{2} x_{1}-u_{2}, \quad x_{3}=\frac{1}{2} x_{2}-u_{3}, \quad x_{j} \geq 0, \quad u_{j} \geq 0 \quad(j=1,2,3),
$$


где $m=1, n=3, a=x_{1}+2 x_{2}+4 x_{3}, f(a)=3 a /(1+a)$. Здесь $q(u)=3 u_{1}+4 u_{2}+4 u_{3}$, $\mu(a)=9 a /(1+a)-a, a^{*}=2, \mu^{*}=4$.

Очевидно, все предположения утверждения 4 выполнены; более того, согласно (20) отображение $F$ здесь является глобально неразложимым. Множество сохраняющих управлений $D^{\prime}$ лежит на плоскости $3 u_{1}+4 u_{2}+4 u_{3}=4$. Поскольку $I^{*}=\{1\}$, находим

$$
V_{D^{\prime}}=\left\{v_{1}\right\}, v_{1}=(4 / 3,0,0), \quad V_{D^{\prime \prime}}=\left\{v_{2}, v_{3}\right\}, v_{2}=(1,0,1 / 4), v_{3}=(0,1,0),
$$

и представление (34) заключения (2) утверждения 3 имеет вид равенства $u=\alpha v_{1}+\beta v_{2}+\gamma v_{3}$, где $\alpha+\beta+\gamma=1, \alpha>0, \beta \geq 0, \gamma \geq 0$. Таким образом, множество $D^{\prime}$ в данном случае - треугольник без одной из сторон (отрезок, соединяющий точки $v_{2}, v_{3}$ уже принадлежит множеству $D^{\prime \prime}$ в силу его замкнутости; см. заключение (1) утверждения 3$)$.

Пусть теперь $c(u)=2 u_{1}+u_{2}+u_{3}$. Тогда $\gamma_{1}=2 / 3, \gamma_{2}=1 / 4, \gamma_{3}=1 / 4$ и $\gamma=2 / 3=\gamma_{1}$. Согласно утверждению 4 это означает, что существует оптимальное сохраняющее управление $\tilde{u}$. Очевидно, $\tilde{u}=v_{1}$; соответствующий вектор состояния положителен: $\tilde{x}=(2 / 3,1 / 3,1 / 6)$.

\section{Заключение}

Одной из важнейших функций моделей устойчивой эксплуатации возобновляемых ресурсов является прогнозирование зависимости состояния эксплуатируемой системы от степени эксплуатации, позволяющее избежать режима сверхэксплуатации, приводящего к деградации системы. В данной работе в довольно общей постановке, когда свойства системы задаются свойствами оператора перехода из предыдущего состояния в следующее, удалось получить свойства этой зависимости (утверждение 1). Кроме того, полученные свойства допустимого множества дают возможность анализа, будет ли оптимальное управление сохраняющим или, возможно, только квазисохраняющим. Так, согласно утверждению 3 в случае $I^{*}=\varnothing$ множество сохраняющих управлений на положительной границе допустимого множества является открытым (см. равенство (35)), и оптимальный вектор может быть только квазисохраняющим (за исключением единственного случая ориентации целевого вектора).

Наконец, получен конструктивный критерий существования сохраняющего оптимального управления (утверждение 4). Перечисленные результаты открывают возможности для построения эффективного алгоритма решения рассматриваемой задачи оптимизации, учитывающего ее особенности в случае использования бинарной модели Лесли.

\section{СПИСОК ЛИТЕРАТУРЫ}

1. The state of world fisheries and aquaculture 2018. Meeting the sustainable development goals. FAO-2018. Rome: UNO, 2018. 210 p.

2. The state of the world's forests 2018. Meeting the sustainable development goals. FAO-2018. Rome: UNO, 2018. 118 p.

3. Wallmo K., Bisack K. D., Lew D. K., Squires D. E. Editorial: The Economics of protected marine species: Concepts in research and management // Front. Mar. Sci. 2016. Vol. 3. P. 1-2. doi: 10.3389 /fmars.2016.00183 .

4. De Lara M., Doyen L. Sustainable management of natural resources. Mathematical models and methods. Berlin; Heidelberg: Springer-Verlag, 2008. 266 p. doi: 10.1007/978-3-540-79074-7.

5. Usher M. B. A matrix approach to the management of renewable resources, with special reference to selection forests // J. Appl. Ecol. 1966. Vol. 3, no. 2. P. 355-367. doi: 10.2307/2401258.

6. Williamson M.H. Introducing students to the concepts of population dynamics // Proc. British Ecological Society symposium "The teaching of ecology". Oxford: Blackwell, 1967. P. 169-175.

7. Dunkel G. M. Maximum sustainable yields // SIAM J. Appl. Math. 1970. Vol. 19, no. 2. P. 367-378. doi: $10.1137 / 0119035$.

8. Doubleday W. G. Harvesting in matrix population model // Biometrics. Vol. 31, no. 1. 1975. P. 189-200. doi: $10.2307 / 2529719$. 
9. Reed W.J. Optimum age-specific harvesting in a nonlinear population model // Biometrics. 1980. Vol. 36, no. 4. P. 579-593. doi: 10.2307/2556112.

10. Getz W. M. The ultimate-sustainable-yield problem in nonlinear age-structured populations // Math. Biosci. 1980. Vol. 48, no. 3-4. P. 279-292. doi: 10.1016/0025-5564(80)90062-0 .

11. Grey D. R. Harvesting under density-dependent mortality and fecundity // J. Math. Biol. 1988. Vol. 26, no. 2. P. 193-197. doi: 10.1007/BF00277732.

12. Getz W. M., Haight R. G. Population harvesting: Demographic models of fish, forest and animal resources. Princeton: Princeton University Press, New Jersey, 1989. 391 p.

13. Caswell H. Matrix population models: Construction, analysis, and interpretation. 2nd ed. Sunderland: Sinauer Associates, Inc. Publishers, 2001. 722 p.

14. Lefkovitch L.P. The study of population growth in organisms grouped by stages // Biometrics. 1965 . Vol. 21, no. 1. P. 1-18. doi: 10.2307/2528348.

15. Caswell H., de Vries C., Hartemink N., Roth G., van Daalen S. F. Age-stage classified demographic analysis: a comprehensive approach // Ecological Monographs. 2018. Vol. 88, no. 4. P. 560-584. doi: 10.1002/ecm.1306 .

16. Goodman L. A. The analysis of population growth when the birth and death rates depend upon several factors // Biometrics. 1969. Vol. 25, no. 4. P. 659-681. doi: 10.2307/2528566.

17. Rogers A. Applied multiregional demography: migration and population redistribution. Cham: Springer, 114 p. 2015. doi: 10.1007/978-3-319-22318-6.

18. Фрисман Е.Я., Кулаков М.П., Ревуцкая О. Л., Жданова О. Л., Неверова Г.П. Основные направления и обзор современного состояния исследований динамики структурированных и взаимодействующих популяций // Компьютерные исследования и моделирование. 2019. Т. 11, № 1 . С. 119-151. doi: 10.20537/2076-7633-2019-11-1-119-151.

19. Смирнов А. И. О некоторых нелинейных обобщениях модели Лесли, учитывающих эффект насыщения // Вестник УИЭУиП. 2010. №4(13). С. 98-101.

20. Mazurov Vl. D., Smirnov A. I. On the reduction of the optimal non-destructive system exploitation problem to the mathematical programming problem // Proc. of VIII International Conf. on Optimization and Applications (OPTIMA-2017) / eds. Yu. G. Evtushenko et al. 2017. P. 392-398.

21. Mazurov Vl. D., Smirnov A. I. Properties of admissible set of an optimal non-destructive system exploitation problem in some general formalization // Proc. of the School-Seminar on Optimization Problems and their Applications (OPTA-SCL 2018) / eds. S. Belim S. et al. Omsk, 2018. P. 359-371.

22. Smirnov A.I., Mazurov Vl.D. On existence of optimal non-destructive controls for ecosystem exploitation problem applied to a generalization of Leslie model // Proc. of IX Internat. Conf. on Optimization and Applications (OPTIMA-2018) / eds. Yu. G. Evtushenko et al. (Supplementary Volume). 2018. P. 199-213. doi: 10.12783/dtcse/optim2018/27933.

23. Рокафеллар Р. Выпуклый анализ. М.: Мир, 1973. 469 с.

24. Никайдо Х. Выпуклые структуры и математическая экономика. М.: Мир, 1972. 518 с.

25. Smirnov A.I., Mazurov Vl. D. Generalization of controls bimodality property in the optimal exploitation problem for ecological population with binary structure // Proc. Internat. Conf. on Optimization and Applications (OPTIMA 2019): Optimization and Applications / eds. M. Jaćimović et al. 2020. P. 206-221. (Ser. Communications in Computer and Information Science; vol. 1145.) doi: $10.1007 / 978-3-030-38603-0 \_16$.

Поступила 11.06.2020

После доработки 20.07.2020

Принята к публикации 24.07.2020

Мазуров Владимир Данилович

д-р физ.-мат. наук, ведущий науч. сотрудник

Институт математики и механики им. Н. Н. Красовского УрО РАН

г. Екатеринбург

e-mail: mazurov@imm.uran.ru

Смирнов Александр Иванович

канд. физ.-мат. наук, старший науч. сотрудник

Институт математики и механики им. Н. Н. Красовского УрО РАН

г. Екатеринбург

e-mail: asmi@imm.uran.ru 


\section{REFERENCES}

1. The state of world fisheries and aquaculture 2018. Meeting the sustainable development goals. FAO-2018. Rome: UNO, 2018, 210 p. ISBN: 978-92-5-130562-1.

2. The state of the world's forests 2018. Meeting the sustainable development goals. FAO-2018. Rome: UNO, 2018, 118 p. ISBN: 978-92-5-130561-4.

3. Wallmo K., Bisack K.D., Lew D.K., Squires D.E. Editorial: The Economics of protected marine species: Concepts in research and management. Front. Mar. Sci., 2016, vol. 3, art.-no. 183, 2 p. doi: 10.3389/fmars.2016.00183 .

4. De Lara M., Doyen L. Sustainable management of natural resources: Mathematical models and methods. Berlin; Heidelberg: Springer-Verlag, 2008, 266 p. doi: 10.1007/978-3-540-79074-7.

5. Usher M.B. A matrix approach to the management of renewable resources, with special reference to selection forests. J. Appl. Ecol., 1966, vol. 3, no. 2, pp. 355-367. doi: 10.2307/2401258.

6. Williamson M.H. Introducing students to the concepts of population dynamics. In: J.M. Lambert (ed.) Proc. of the British Ecological Society symposium The Teaching of Ecology. Oxford: Blackwell, 1967, pp. 169-175. ISBN: 978-0632025701.

7. Dunkel G.M. Maximum sustainable yields. SIAM J. Appl. Math., 1970, vol. 19, no. 2, pp. $367-378$. doi: $10.1137 / 0119035$.

8. Doubleday W.G. Harvesting in matrix population model. Biometrics, 1975, vol. 31, no. 1, pp. $189-200$. doi: $10.2307 / 2529719$.

9. Reed W.J. Optimum age-specific harvesting in a nonlinear population model. Biometrics, 1980, vol. 36, no. 4, pp. 579-593. doi: 10.2307/2556112.

10. Getz W.M. The ultimate-sustainable-yield problem in nonlinear age-structured populations. Math. Biosci., 1980, vol. 48, no. 3-4, pp. 279-292. doi: 10.1016/0025-5564(80)90062-0 .

11. Grey D.R. Harvesting under density-dependent mortality and fecundity. J. Math. Biol., 1988, vol. 26, no. 2, pp. 193-197. doi: 10.1007/BF00277732.

12. Getz W.M., Haight R.G. Population harvesting: Demographic models of fish,forest, and animal resources. Princeton, New Jersey: Princeton University Press, 1989, 391 p. ISBN: 9780691085166.

13. Caswell H. Matrix population models: Construction, analysis, and interpretation. 2nd ed. Sunderland, Massachusetts: Sinauer Associates, Inc. Publishers, 2001, 722 p. ISBN: 0-87893-096-5.

14. Lefkovitch L.P. The study of population growth in organisms grouped by stages. Biometrics, 1965, vol. 21, no. 1, pp. 1-18. doi: 10.2307/2528348.

15. Caswell H., de Vries C., Hartemink N., Roth G., van Daalen S.F. Age-stage classified demographic analysis: a comprehensive approach. Ecological Monographs, 2018, vol. 88, no. 4, pp. 560-584. doi: $10.1002 /$ ecm.1306 .

16. Goodman L.A. The analysis of population growth when the birth and death rates depend upon several factors. Biometrics, 1969, vol. 25, no. 4, pp. 659-681. doi: 10.2307/2528566.

17. Rogers A. Applied Multiregional Demography: Migration and Population Redistribution. Cham: Springer, 2015, 114 p. doi: 10.1007/978-3-319-22318-6 .

18. Frisman E.Ya., Kulakov M.P., Revutskaya O.L., Zhdanova O.L., Neverova G.P. The key approaches and review of current researches on dynamics of structured and interacting populations. Computer Research and Modeling, 2019, vol. 11, no. 1, pp. 119-151 (in Russian). doi: 10.20537/2076-7633-2019-11-1-119-151.

19. Smirnov A.I. On some nonlinear generalization of the Leslie model considering the effect of saturation. Vestnik Ural'skogo Instituta Ekonomiki, Upravleniya i Prava, 2010, no. 4 (13), pp. 98-101 (in Russian).

20. Mazurov V.D., Smirnov A.I. On the reduction of the optimal non-destructive system exploitation problem to the mathematical programming problem. In: Yu.G. Evtushenko, M.Yu. Khachay, O.V. Khamisov, Yu.A. Kochetov, V.U. Malkova, M.A. Posypkin (eds). Proc. of VIII Internat. Conf. on Optimization and Applications (OPTIMA-2017), 2017, pp. 392-398.

21. Mazurov V.D., Smirnov A.I. Properties of admissible set of an optimal non-destructive system exploitation problem in some general formalization. In: S. Belim, A. Kononov, Yu. Kovalenko (eds). Proc. of the School-Seminar on Optimization Problems and their Applications (OPTA-SCL 2018). Omsk, Russia, 2018, pp. 359-371.

22. Smirnov A.I., Mazurov V.D. On existence of optimal non-destructive controls for ecosystem exploitation problem applied to a generalization of Leslie model. In: Yu.G. Evtushenko, M. Jaćimović, M.Yu. Khachay et al. (eds). Proc. of IX Internat. Conf. on Optimization and Applications (OPTIMA2018) (Supplementary Volume), DEStech Transactions on Computer Science and Engineering, 2018, pp. 199-213. doi: 10.12783/dtcse/optim2018/27933. 
23. Rockafellar R. Convex Analysis. Princeton: Princeton University Press, 1970, 451 p. ISBN: 0691015864. Translated to Russian under the title Vypuklyi analiz. Moscow: Mir Publ., 1973, 469 p.

24. Nikaido H. Convex Structures and Economic Theory. New York: Academic Press, 1968,405 p. ISBN: 9781483230030. Translated to Russian under the title Vypuklye struktury i matematicheskaya ekonomika. Moscow: Mir Publ., 1972, 518 p.

25. Mazurov V.D., Smirnov A.I. Generalization of Controls Bimodality Property in the Optimal Exploitation Problem for Ecological Population with Binary Structure. In: M. Jaćimović, M. Khachay, V. Malkova, M. Posypkin (eds), Optimization and Applications, 2020, Communications in Computer and Information Science, vol. 1145, Cham: Springer, pp. 206-221. doi: 10.1007/978-3-030-38603-0_16.

Received June 11, 2020

Revised July 20, 2020

Accepted July 24, 2020

Funding Agency: This work was supported by the Russian Foundation for Basic Research (project no. 19-07-01243).

Vladimir Danilovich Mazurov, Dr. Phys.-Math. Sci. Krasovskii Institute of Mathematics and Mechanics of the Ural Branch of the Russian Academy of Sciences, Yekaterinburg, 620108 Russia, e-mail: mazurov@imm.uran.ru.

Aleksandr Ivanovich Smirnov, Cand. Sci. (Phys.-Math.), Krasovskii Institute of Mathematics and Mechanics of the Ural Branch of the Russian Academy of Sciences, Yekaterinburg, 620108 Russia, e-mail: asmi@imm.uran.ru .

Vl. D. Mazurov, A. I. Smirnov. A criterion for the existence of nondestructive controls in the problem of optimal exploitation of an ecosystem with a binary structure, Trudy Instituta Matematiki i Mekhaniki URO RAN, 2020, vol. 26, no. 3, pp. 101-117. 